\title{
Demographic Processes and Ethnic Residential Segregation
}

\author{
ITZHAK OMER* \\ Department of Geography, Tel Aviv University, Tel Aviv 69978, Israel
}

(Received 16 November 1998)

\begin{abstract}
This paper examines the involvement of demographic processes in the residential segregation of ethnic groups in an urban area. For this purpose, a two-part methodology has been employed. The first part comprises a conceptual framework which uses the concept of timespace resources as an analytical tool to clarify the structural constraints embedded in the changes in ethnic residential distribution. The second part comprises a simulation model of ethnic residential segregation. The model, which fits into the category of agent-based simulation models, serves as a tool for heuristic study. The methodology has been applied to examine the implications of fluctuations in ethnic group diffusion and the residential mobility rate on the majority-minority residential segregation, and on the internal ethnic segregation within the minority group.
\end{abstract}

Keywords: Ethnicity, Residential segregation, Demography, Agent based simulation, Time-geography

\section{INTRODUCTION}

The vehicles for residential segregation are residential mobility and form of social group diffusion in an urban area. However, their nature derives not only from spontaneous and goal directed actions, but also from urban processes that are not directly related to inter-group relations, such as apartments inventory, the structure of the built-up urban environment, and as will be shown here, demographic processes. The meaning is that residential mobility serves as a means of involvement of various forces in the design of residential segregation.
This situation presents a methodological problem: How to distinguish between the various forces and evaluation of the intensity and character of their influence? Nevertheless, even if we overcome this problem, another substantial problem is presented: How is residential segregation influenced by residential mobility changes such as changes in the rate of residential mobility or fluctuations in the diffusion rate of minority group?

The fundamental difficulty derives from the attempt to deal with the immanent components of the residential segregation process, which have their own internal relations, in the sense that they create

*Tel.: +973(3)6406841. Fax: +972(3)6406243. E-mail: omery@post.tau.ac.il. 
one another. Overcoming the problematic aspects of the fundamental issues pertaining to demographic and social changes, requires a methodology that allows evaluation of components and differentiating between them by abstracting from real life situations, as presented in the realism method (Sayer, 1984). The attempt to differentiate between the components and address some of them is a purely heuristic device, and naturally does not indicate a standpoint about the nature of the relationship between the components. The conceptual framework must be accompanied by a means for heuristic examination of the relationship investigated here at the operative level as well. In addition to enabling abstraction of the components involved in the social process, heuristic examination also enables assumptions to be made, including those contrary to the fact, in order to evaluate how components and events operate, as well as their contribution to the development of residential segregation.

The present methodology combines a conceptual framework and a heuristic simulation model. It is intended to facilitate understanding of the structural relations between demographic changes that occur during the diffusion of a minority group's spatial pattern, as well as the study of majorityminority segregation and segregation within minority groups. The article is structured as follows. The first part presents the conceptual framework and a heuristic simulation model. The second part applies the methodology to examine the involvement of demographic processes in residential segregation, and concludes with a discussion of the potential use of the methodology in the study of demographic and social processes.

\section{CONCEPTUAL FRAMEWORK}

Conventional wisdom in the discipline of geography and the philosophy of society is that space and time are not merely a passive framework or an expression of the occurrence of social processes, but are immanent in them (Benno, 1993). For example, space and time are critical components in the explanation of key mechanisms proposed in the structuration theory; they are recruited in order to explain how unintended results and unconscious settings arise from the intentional actions of individuals (Giddens, 1984). The central idea, to which this theory has provided the basis, is that space and time serve as the means and the conditions for the emergence of a social system. It is my assertion that socio-geographic distribution, or residential segregation in the present instance, is not any different from the overall social system. i.e., space and time are not only a means for defining residential distribution (the location of people in space and time); they are also the means for its evolution. Through them, the actions of individuals participating in the emerging residential distribution have unintended results and residential distribution takes place under cognizant conditions enabling its internal organization which provides its stability. The difference between geographic distribution and social form lies in its being defined, among others, according to objects that have a material dimension. This difference gives it a degree of autonomy with respect to social form, and thereby, involvement in its formation (Omer, 1996).

Before attempting to clarify how space and time can be used as tools for understanding residential segregation, the term material dimension should first be placed in the relevant conceptual context. In geographic and social research as a rule, the focus on space and time is associated primarily with time geography, used to formulate an explanation for the constraints on individuals' actions in terms of space and time (Hagerstrand, 1970). The idea that physical space and time constrain any object that has a material dimension is used as a basis to discuss the involvement of space-time constraints both in shaping day-to-day activities and with respect to the group level and the uni-directional movements associated with the life cycle of individuals. Time geography has highlighted the vital need to address space-time issues in models of residential choice (Flowerdew, 1978), and in analyzing processes of social and demographic change. Carlstein (1982), 
for example, used space and time resource constraints to examine how changes in carrying capacity resulting from technological and environmental developments bring about social and demographic changes in a variety of cultures.

Resting on this conceptual work, a conceptual framework has been formulated here to locate structural constraints on the actions of individuals participating in the residential segregation process as well as structural constraints on the spatial pattern diffusion of ethnic minority in the city. Locating structural constraints will facilitate tools for examining the influences of the residential mobility processes involved in shaping the residential spatial pattern of social groups.

\subsection{Structural Constraints on the Actions of Individuals}

The participants in the residence segregation process are faced with a variety of constraints. These include capability constraints, expressed in moving residence due to economic pressures, and the frequency of such moves; the number of times individuals change their place of residence is limited. There are also coupling constraints; change of place of residence requires at least two elements (vacating and occupying). Finally, they also encounter two types of authority constraints. One derives from the voluntary social establishment, which limits the choices open to social groups; the other derives from institutional intervention, such as the municipal authority preventing one of the populations from choosing a place of residence in certain parts of the urban area, or directly influencing residential mobility. These three constraints, which restrict and define space and time resources, are bound together in the concepts of packing capacity and carrying capacity, expressed in terms of the restricted space-time resources assigned to a collection of individuals to carry out a project. Applying such terminology, the formation of residential distribution can be said to be the execution of a project that requires time-space resources.
Despite the fact that these constraints are prerequisites, the degree of their intensity and influence depends on the actions of individuals. The space resource - defined as feasible residential space, and the time resource - defined in terms of the frequency of residential mobility, are structural constraints on action. They serve as means and conditions for the social activity that occurs within their framework. At the same time, the actions of individuals may intensify or diminish them. The feasible residential space, for example, is dependent on the timing of the residential mobility, which is the product of the actions of individuals. That is, the intensity of the constraint depends on how space and time resources are utilized. According to this distinction, one may differentiate between given constraints to action, and constraints as a consequence of actions. Structural constraints to action are constraints that impact on the space and time resources before they are used. Structural constraints as a consequence of actions are constraints stemming from the way in which space and time resources are utilized.

\subsection{Structural Constraints on the "Action" of Residential Spatial Pattern}

In order to evaluate the involvement of demographic processes in residential segregation, it is necessary to locate structural constraints comprised in the residential spatial pattern diffusion. These are the constraints to the "action" of the residential spatial pattern, and general population movement, as a whole. For this purpose, it is necessary to understand how space and time are bound together and dependent on each other in the process of minority group diffusion in the urban area. In short, the residential segregation process must be translated into concepts of space and time.

It then follows that to examine the properties and components of the residential segregation process which is characterized in spatio-diffusive terms (Woods, 1980), a spatial diffusion model is needed. The diffusion model suggests an explanation for the way the transition from one distribution 
to another occurs. More specifically, the model is designated to address spatial diffusion of a phenomenon or innovation, with the express purpose of concentrating on the morphology of the process (Hagerstrand, 1967). When the morphological formulation is placed at the center of investigation, it opens the possibility to examine a wide range of phenomena. And accordingly, Morrill (1970), in formulating real-life diffusion processes, compared the way a ghetto diffuses with the way other phenomena diffuse, such as epidemics and information, and noted the morphological similarity between them. However, formulating the morphology of minority group diffusion in terms of space and time is not enough. If space and time are to be used as analytical tools, it is necessary to understand how this diffusion works and the structural constraints specific to it.

Despite the morphological similarity to innovation diffusion, minority group diffusion has a unique potential for change, owing to the difference between material and information. Information can exist everywhere, or not at all, while individuals and groups as material entities must exist somewhere at a particular point in time. In other words, population movements encounter space and time constraints. Figure 1 illustrates this difference. In the case of the innovation diffusion, forms $\mathrm{A}$ and $\mathrm{B}$ are possible in the same area, and one may even crossover to the other, in accordance with the nature of the innovation and the properties of the potential population to accept it. However, in the case of minority group diffusion, crossover from form $\mathrm{A}$ to form $\mathrm{B}$ or vice versa is not possible unless there is a change in the invasion rate of the minority population into the area inhabited by the majority. The restrictive nature of the material dimension manifests itself in the relationship between two components: the size of the geographical area, in which the change in minority/majority population composition is occurring, and the rate of the change in the population composition in each unit in this area. That is, expansion of the geographical area, in which the change in population composition is taking place within a given time, necessitates a change in the rate of population composition change of each unit, and vice versa. A transition from the geographic area size of form A to that of form B (see Fig. 1) by necessity dictates a transition to a slower rate of population composition change of each geographic unit, as demonstrated in form C.

In order to clarify the dependent relations between the rate of change of the population composition in a geographic unit and the size of the geographic unit, and its implications on the degree of residential segregation, Fig. 2 presents three ideal forms of minority group diffusion in a geographic area, comprising three units. The forms are differentiated by the size of the geographic area in which the change in ethnic composition occurs. In form $\mathrm{A}$, the change in the ethnic composition takes place in one geographic unit, in form B two units, and in form $\mathrm{C}$ three units. Moreover, according to the rule by which a change in one of
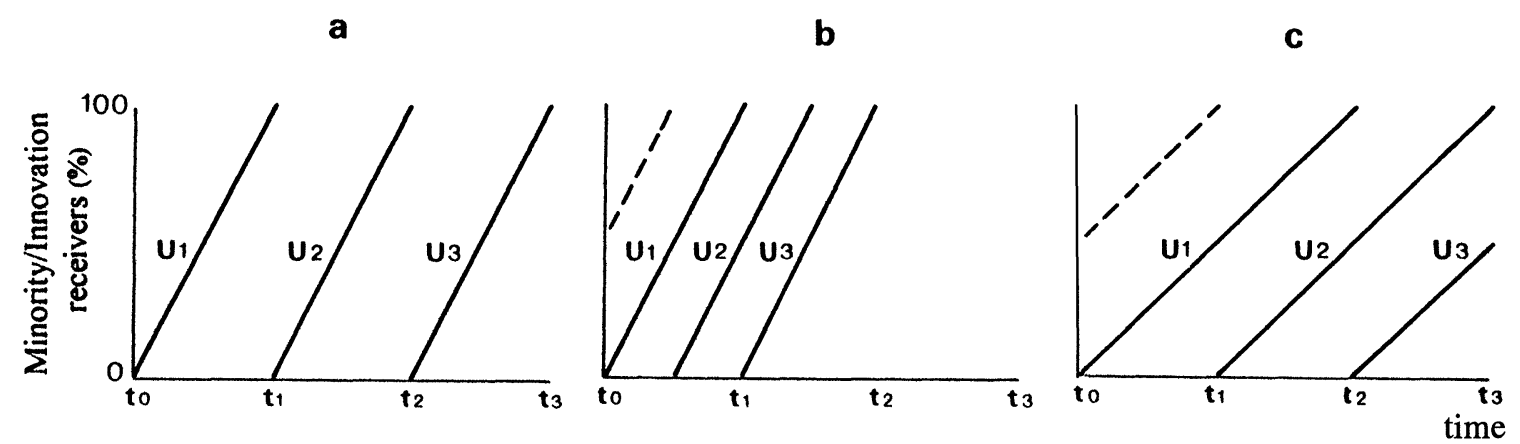

FIGURE 1 Minority group diffusion versus innovation diffusion. $Y$-axis: The percentage of the minority population/receivers of the innovation in a geographic unit; $X$-axis: Time; $\mathrm{u} 1, \mathrm{u} 2, \mathrm{u} 3$ - geographic units. 
a

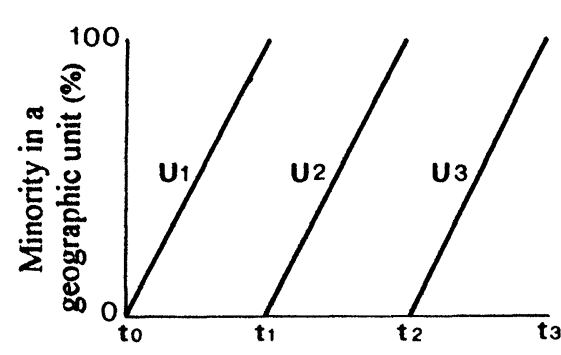

b
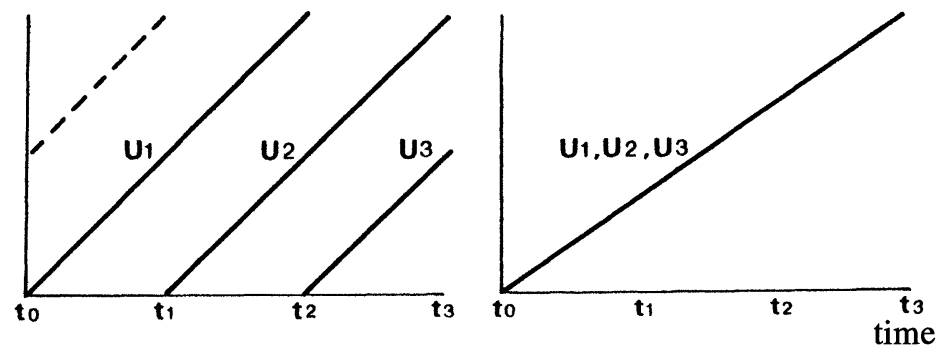

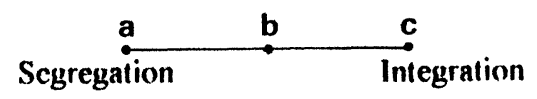

FIGURE 2 Minority group diffusion and residential segregation - the relation between the geographic space and the rate of change of population majority-minority composition.

the components necessitates a change in another, the form must be differentiated by the rate of change in each geographic unit. The difference between the forms reflects the difference in the degree of residential segregation between social groups and the exposure time of each member of one group to the members of the other group. That is, if the minority population settles over a greater area (and accordingly the rate of change per unit is slower), the degree of segregation must be weaker, and vice versa. This description shows that the degree of residential segregation and the form of the residential spatial pattern diffusion of a minority can be defined by the same components, and therefore have the same structural constraint.

The structural relationship presented above is also applicable to the examination of the internal differences within a minority group. Since ethnic variation within a minority group occurs within the framework of the spatial pattern of the minority group, it is affected by the changes within it. Effectively, the shape of the spatial pattern diffusion of a minority group, or the degree of segregation from the majority group, defines the feasible residential space, in which the internal ethnic variance can express itself.

To enable the use of this methodology in the operative examination of the broad range of processes involved in shaping ethnic residential distribution, a simulation model has been built as a heuristic study tool, and is described below.

\section{AGENT-BASED SIMULATION OF RESIDENTIAL SEGREGATION}

The model built belongs to the general category of cellular space (CS) models, which have been used in a wide range of scientific fields, including geographic research. CS models were built as a means for studying the dynamics of human geographic phenomena, such as the spatial pattern diffusion of a minority group in an urban area (Morrill, 1965). However, it was Tobler (1979) and Couclelis (1985; 1988) who first explicitly discussed the potential and possible uses of CS models in investigating geographic phenomena, emphasizing the inherent advantages of their spatial nature and their indispensability in examining spatial patterns in the process of formulating a theoretical explanation.

CS models that deal with the evolution of complex systems and self-organization processes by focusing on the relationship between micro-level and macro-level phenomena can be divided into two categories: cellular automata (CA) models and agent-based simulation (ABS) models. CA models have been applied in studies that aim to identify the basic principles of self-organization in the course of the development of spatial patterns in a diverse 
range of scientific disciplines, including biology and neurobiology (Demongeot et al., 1985), biochemistry (Langton, 1986) and physics (Mannevill et al., 1989). In the field of geography, such models have been used as heuristic platforms (Couclesis, 1988; Phipps, 1989; Portugali et al., 1994), and as means of dealing with real life patterns (Wu, 1996; White et al., 1997).

CA models enable the examination of urban processes, focusing on urban infrastructure such as land-use represented by means of cellular space, with the cells representing, for example, areas in the city (micro-level) and the cellular space of the city as a whole (macro-level). In ABS models, on the other hand, the focus is on human behavior in the urban space, also represented as cellular space, and the models are capable of representing a large number of participants - multi-agent simulation. It is therefore the behavior of agents (rather than areas in the urban space) that constitutes the micro-level, and the population distribution that constitutes the macro-level. This feature enables explicit examination of the behavior of agents in the geographic area, and in particular, residential mobility and the evolving of the population distribution (Openshow, 1995; Portugali et al., 1997; Benenson and Portugali, 1997). In this type of model "We can frame our hypothesis at the individual level, directly in terms of individual preferences, abilities, and imperfect information, and explore the implication of each for the patterns and properties that emerge at the macro levels" (Dibble, 1996, p. 211).

The multi-agent simulation version of the ABS model built here is designed to enable the heuristic study of residential ethnic segregation, with explicit reference to human behavior and traits, constraints encountered by individuals, and the properties of the residential distribution evolving in the residential segregation process between ethnic groups. The model structure and features are described by way of the main working screen displayed while the user views the simulation (Fig. 3). The screen displays the cellular space and the residential distribution moving across it. The features and properties of the model are presented below.

\subsection{Model Structure}

Representation of agents with ethnic identities The cellular space (the black grid) represents a physical geographic area and each cell in it simulates a home capable of containing a nuclear family or being empty. The generations of the simulation represent standard calendar periods such as years. In the simulation output presented in Fig. 3, the cellular space is defined as $30 \times 30$. The cellular space is divided into discrete units using a predefined set of cells (the yellow grid). These units represent the places targeted as residential choices. This setting reflects the stand that the socio-urban space, at least with respect to ethnic relations which are based on solidarity and a sense of identification, is not a continuous field, but discrete in essence. In this context, the model simulates diffusive distribution of a minority group into the residential area of a majority population. For this reason, the simulation is initialized with families from the majority group "residing" in the cell space (the white cells). Minority group families then diffuse into this space. To address the internal variance within a minority group, it should be noted that ethnicity in the present context represents an attribute such as: race, religion, nationality, or a combination of these attributes, which creates vertical division in the social system (Boal, 1987). Thus, to an agent living in a complex ethnic system one can assign an ethnic set comprising ethnic identities nested within one another, according to the ethnic groups to which the agent belongs (Hendlman, 1977). Accordingly, each family entering the area, represented by a unit that has a memory moving in the cellular space, has a set of ethnic identities associated with it. The level of complexity of an "incoming" family's identity may be defined in a variety of ways, depending on the subject under investigation.

In this situation, where each family is represented by a mobile unit that "remembers" a set of identities that are fully nested, each family is simultaneously associated with several residential distributions, and accordingly, participates in the creation of spatial 


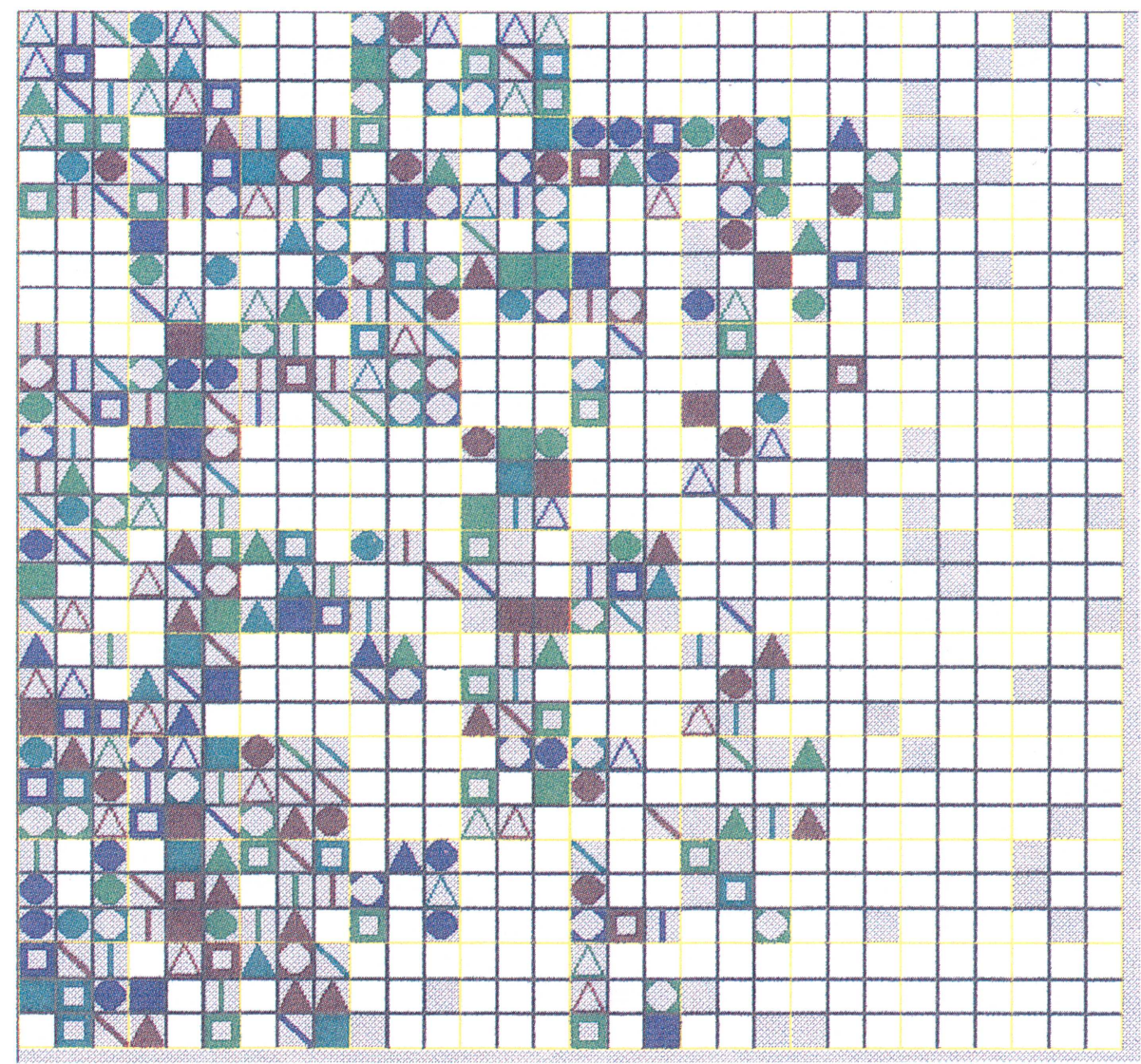

FIGURE 3 Multi-agent simulation main screen. The cellular space depicted here shows the emergence of a minority residential spatial pattern (20th generation of the simulation), based on three attributes: community, sub-community and extended family. There are four communities, eight sub-communities (two sub-communities per community) and 32 extended families (four extended families per sub-community); every group of cells with the same markings (color and shape) comprises one extended family. (See Color Plate VI.)

patterns of family, community, etc. Formally stated: the model simulates residential distribution, based on a set of attributes. Let us assume the existence of three attributes, differentiated by level of generality: L1, L2 and L3. Attribute L1 is the most general, L2 less so, and L3 the least. The attributes define a set of identities for each family entering the cell space. If $i, j, k$ represent a family's set of identities, then the following statement can be made: $\{i, j, k\} \mid i=1 \ldots n 1 \in \mathrm{L} 1, j=1 \ldots n 2 \in \mathrm{L} 2$, $k=1 \ldots n \in \mathrm{L} 3$. After the family has entered the cellular space, it is defined at every stage of the simulation as follows: $P_{x, y}(i, j, k)$, where $x$ and $y$ indicate the family's location in the cellular space, and $i, j, k$ represent the ethnic identities which are fully nested $k \subset j \subset i$. From this definition, it can be stated that the distribution defined according to a general attribute contains the distribution defined according to the less general attribute: $\sum P_{x, y}(i) \subset$ $\sum P_{x, y}(j) \subset \sum P_{x, y}(k)$.

Choosing a place of residence A family's search for a place of residence is based on the principle of "proceeding along the identity axis". A family prefers to live in proximity to similar families or to those with similar ethnic identity. The search algorithm of "proceeding along the identity axis" is formulated according to satisficer behavior, a procedure of choosing, one of the ideal types of choosing in a social system (Lierop, 1986, pp. 100-101). According to the procedure, 
a family chooses the one place out of many possibilities that satisfies its requirements e.g., "desire to live in a place that has at least two nuclear families with the same community identity".

Spatial diffusion forms The general form of the process is determined by two factors: a condition that only permits a minority group to enter a particular place if a minority group has already entered places nearby; and a restriction limiting the commencement of diffusion to the left column of places in the cellular space. The more specific form of the process is determined by a tolerance curve, which defines the rate of desertion by the majority population according to the majority/ minority constellation at every "place" in the cellular space (which represents a geographic unit under examination). This function is based on probabilities.

Measuring segregation To measure the level of segregation in the residential distribution during the simulation, the following segregation indices have been included in the model: Dissimilarity index which measures homogeneity, and $P^{*}$ index which measures exposure. The indices are run in every generation executed in the model.

Each of the above components is defined when the model is initialized: cellular space size, the group of geographic locations on which the ethnic structure simulated in the action is overlaid, and the form of the spatial diffusion. During the simulation, the user may intervene and change certain control parameters, including: rate of minority group diffusion and rate of residential mobility within its spatial pattern.

\subsection{Model Features}

The combination of a deterministic mechanism and voluntarism is in line with models built to examine self-organization in social systems (Portugali et al., 1994). On the one hand, the model includes a "social structure", which dictates the search algorithm for a place of residence, and on the other, it allows expression of free choice and chance. Chance is included in all the elements of an event: the identity of the families wishing to enter the area; the identity of the families moving within the area; where places of residence have been vacated due to their desertion by the majority population; and the place chosen by each of the families as the objective of residential mobility. Furthermore, the very depiction of the system in terms of cellular space enables reference to spatial relations and the structure created with this set of relations. The system state appears directly in each iteration, so that the cellular space is equivalent to phase space, which is generally used to help determine the state of the system (Langton, 1986).

Despite points of similarity with ABS models, and multi-agent simulation models in particular, the proposed model has certain unique features worth mentioning. The first is the way in which space and time are represented, and the ability to define the scale of the space-time resources with the model. This type of model construction is not common in research on the spatial organization of social groups, which tends to represent segregation by means of abstract space (e.g., Weidlich, 1987), and thus without space-time constraints. Moreover, since the model simulates a process involving a large number of participants, it enables representation of space and time not only as a framework within which the segregation process occurs, but as immanent components. That is, representing agents as mobile memory units enables reference not only to structural constraints applying to the residential spatial pattern as a whole, but also to constraints encountered by agents and constraints deriving from the actions of agents. A second feature is that it takes into consideration the multifaceted ethnic identities of the individual and the vertical division of the residential distribution. In the many and diverse applications of the CS model, including the ABS simulation model, it is standard practice to use a system of individuals or components that have a horizontal division as a means of simulation. A third feature pertains to the interaction between the individuals or between cells in the cellular space. "Direct" interaction between cells is not restricted to physical space; each individual has "neighbors" 
that are not necessarily physically adjacent. This distinguishes the present model from other cellular space models in which the action of an object is contingent on the presence of a physical link.

\section{APPLICATION OF THE METHODOLOGY}

The conceptual framework and heuristic simulation model presented above serve as the means for examining the influence of fluctuations in the spatial pattern diffusion rate of minority group and the rate of residential mobility within the pattern on the degree of segregation in the ethnic residential distribution. This examination is conducted individually on spatial pattern diffusion in minority housing, and residential segregation, respectively.

\subsection{Residential Spatial Pattern Diffusion of Minorities}

To study the implications of changes that occur in the spatial pattern diffusion rate of a minority group on the degree of segregation in the majorityminority residential distribution, two mechanisms need to be addressed that offer an explanation of minority group diffusion in an urban area: "invasion succession" and "filtering" (see for example, Godfrey, 1988). The first mechanism assumes that the minority group invades the neighborhoods inhabited by the majority group and dictates the rate at which they are pushed out. The second mechanism assumes that the majority population is the driving force and dictates the spatial pattern diffusion to the minority group, with the minority group merely responding.

To reflect reality as closely as possible, the simulation model should include the two mechanisms which exist in real-life residential segregation. Based on this assumption, the simulation model has been defined in the following manner: the residential segregation process is simulated by a set of identities. The nuclear family, as the most basic unit, is simulated by a memory unit (which retains its ethnic identities) that moves within the cellular space in order to find a place of residence (a cell in the cellular space). The cellular space simulates a geographic area inhabited by the majority group. The minority group enters the area, and is also marked by internal ethnic diversity. In addition to its identity as a minority, each nuclear family also has a community identity and sub-community identity defined for it. There are two communities and four sub-communities. Choice is implemented according to the principle of "proceeding along the identity axis" and is the same for all families (Fig. 4). The search is first made in places of residence (a place of residence consists of a set of nine cells) according to proximity to members of the extended family. If no vacant place is found, the search is then based on proximity to the community. If this too proves unsuccessful, the search is based on the minority group's identity.

In each generation (time step) in the simulation, the following events occur:

- Three percent of all the nuclear families belonging to the minority group enter the cellular space.

- The probability of desertion by a family from the majority population is calculated according to

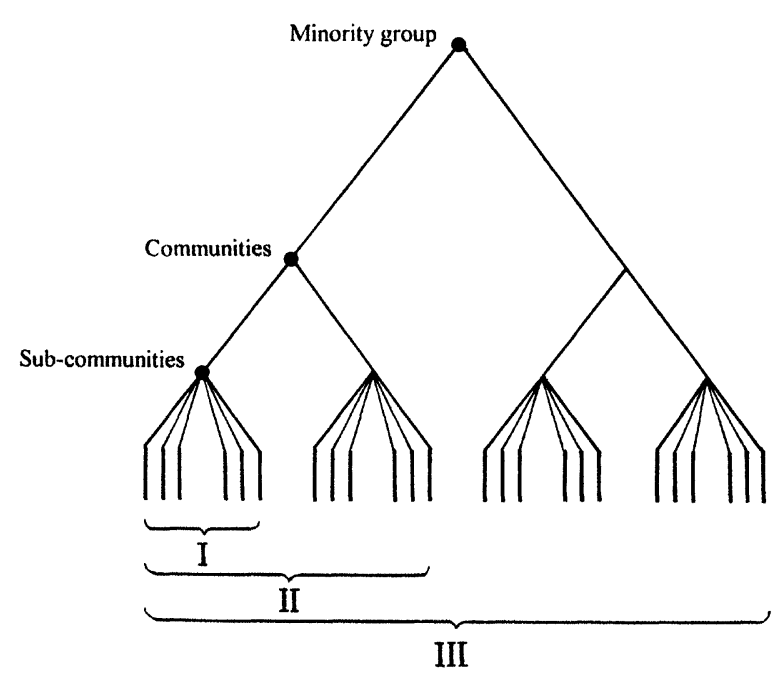

FIGURE 4 Choosing residency according to the principle of proceeding along the identity axis - minority group including two communities and four sub-communities. 
the probability function presented in Fig. 5. According to this function the desertion probability is influenced by the weight of the minority population at the place of residence.

- The probability of residential mobility in a minority group family within the cellular space is $0.1(\mathrm{Mp}=0.1)$ in each generation (one in ten generations).

To examine the influence of fluctuations in the diffusion rate of minority group on the degree of segregation, the model has been applied to compare two simulation processes, differentiated solely by the frequency of changes in the rate of minority group diffusion into a urban area inhabited by a majority population. The total population entering the area over generations $t 0-t 16$ is the same in both instances, as are all the other components in the process. The difference is in the relative division of a given population quantity between generations. In the first process the rate is constant: $3 \%$ of the minority population enter the cellular space in each generation. In the second process, the rate changes according to a fixed sequence $(1 \%, 1 \%, 7 \%)$. Since the diffusion rate is the only difference between the two segregation processes, it is possible to examine the implications of this component on the degree of

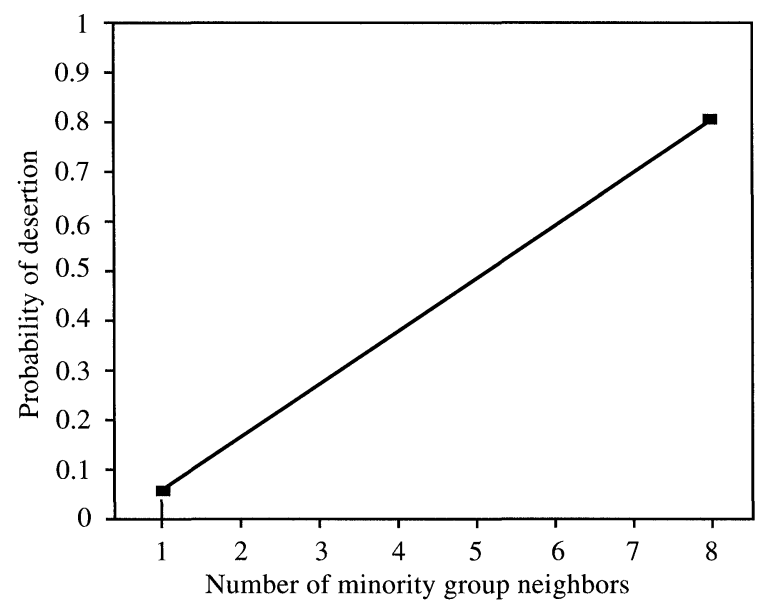

FIGURE 5 Tolerance function - the probability of desertion of the majority population in every place is determined by the number of minority group neighbors (in each place a total of nine cells). segregation between majority and minority populations (Fig. 6(a)), and on the degree of segregation within the minority group (Fig. 6(b)). The results show that the process featuring the constant entry rate creates a residential distribution with greater segregation than the process featuring a variable rate. That is, diffusion rate fluctuations, resulting from many causes, but most probably having nothing to do with the interaction between the groups, have a significant impact on the degree of residential segregation.

To clarify the results, let us look at the structural relationship between the rate of change in the
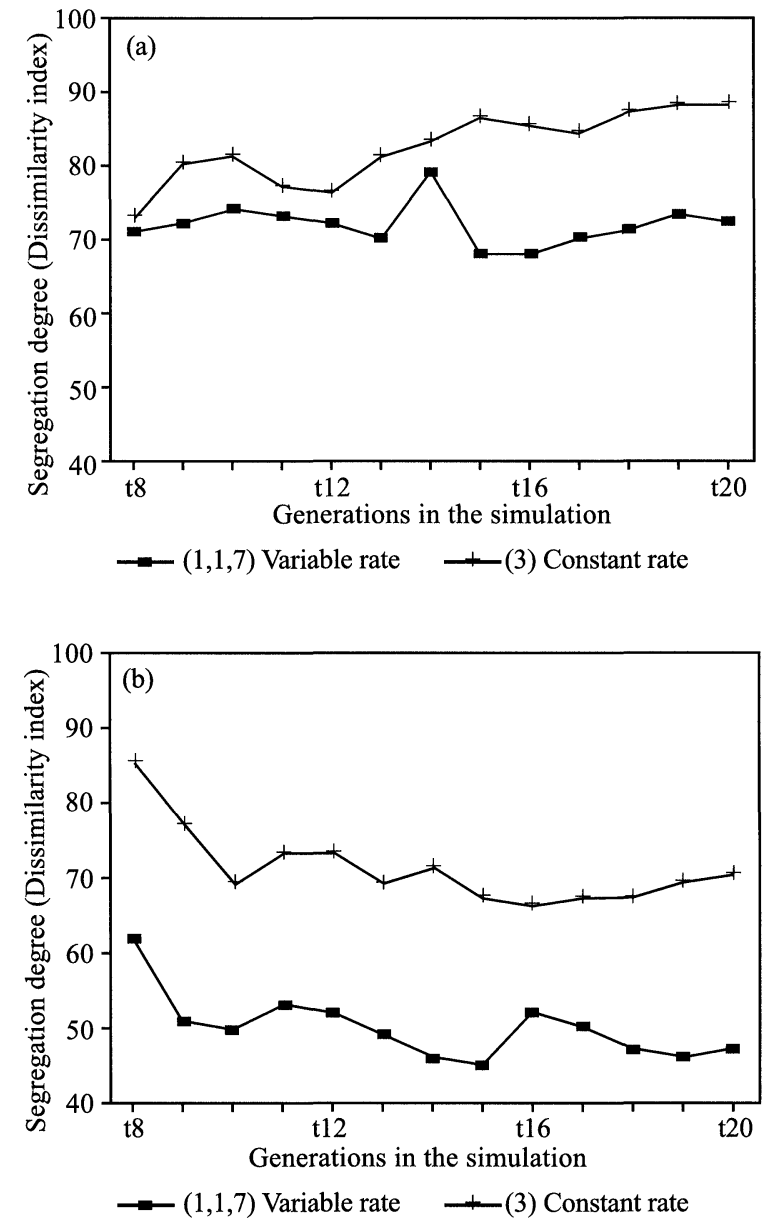

FIGURE 6 The effect of fluctuations in the diffusion rate on residential segregation. (a) The dependence of majorityminority residential segregation on the diffusion rate. (b) The dependence of internal segregation on the diffusion rate. 
majority-minority constellation in each geographic unit and the size of the geographic area in which the change occurs. The rule is that a change in one of the components forces a change in another (changing the rate of transformation in a geographic unit forces expansion or shrinkage of the geographic area in which the change in ethnic constellation is taking place. The opposite is also true). According to this constraint, increasing the quantity of the population entering the cellular space within a specified time frame (in some or all of the generations) constitutes a space-time constraint, in the wake of which the area that the minority population has settled has grown, and this has a direct bearing on the degree of majority/minority segregation. Therefore, when the rate of change in each geographic unit has a certain degree of autonomy, as an expression of the filtering mechanism, then increasing the rate of invasion $(7 \%$ in every generation) must result in the enlargement of the geographic area. And since the increased rate is followed by a slower rate in subsequent generations ( $1 \%$ in each generation), the result is that the mean rate of change in each geographic unit is slower over the whole process, and this is expressed in the degree of segregation in the majority-minority residential distribution. A change in the spatial pattern diffusion rate for the minority group therefore constitutes a structural constraint that necessitates the enlargement of the geographic area in which the change in ethnic composition is occurring, this in turn weakening the residential segregation between majority and minority populations.

Fluctuations in the spatial pattern diffusion rate means that there will be a "mixture" of segregation types in the framework of a single process. The result is that for any spatial pattern diffusion mechanism for minority housing, and in any combination of the mechanisms, a change in the rate of minority group diffusion has implications on residential segregation, whether through a change in the exposure area or the exposure time between the groups.

A comparison of the two simulation processes with respect to internal segregation within the minority group (Fig. 6(b)), shows the strong influence of the spatial pattern diffusion rate of minority groups. This is not surprising. Enlarging the geographic area settled by the minority population means an increase in the space resource utilized by the ethnic groups within the minority groups for segregation among themselves. Increasing the geographic area in which the majority-minority segregation occurs, and thereby weakening it, increases the practical opportunities of minority group members of segregation among themselves.

\subsection{Residential Mobility Rate}

The diffusion rate of residential spatial pattern is relevant to both the majority-minority residential distribution and internal segregation within the minority group. In contrast, residential mobility within the residential spatial pattern is particularly relevant to internal segregation. Residential mobility occurs within a given geographic area, so that its rate (the frequency of the change of residence) influences the extent of the time resource. A time constraint means a limit on the number of times residential mobility may occur, and therefore a change in its rate means either a reduction or increase in the time resource. A relatively high mobility rate means a weakening of the capacity constraints on the agents participating in the process, and resulting in an increase in the time resource available to the groups in the course of the residential segregation process; a high rate means an increase in the opportunities available to the agent, and at the same time, a chance to acquire the place of residence that matches his inclinations. This can be easily demonstrated by comparing the three processes differentiated by the rate of residential mobility: in the first process, there is no mobility at all and the rate is $0(\mathrm{Mp}=0$; the probability of changing residency per generation); in the second, the mobility rate is $\mathrm{Mp}=0.3$ per generation; and in the third $\mathrm{Mp}=0.6$ per generation. When reviewing the degree of segregation during the simulation (Fig. 7(a)), two important issues can be noted: increasing the residential mobility rate leads to an 

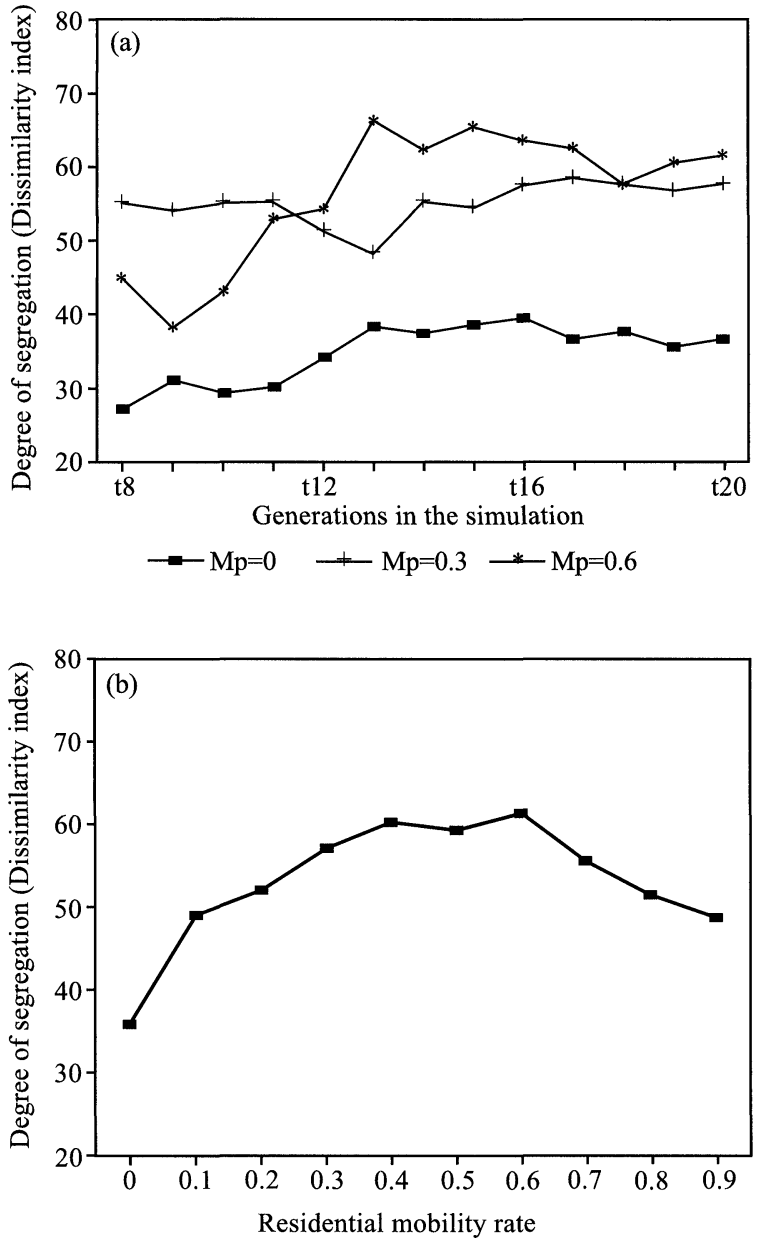

FIGURE 7 The effect of changes in the rate of residential mobility on residential segregation. (a) The degree of internal segregation throughout time as a function of the rate of residential mobility. (b) The degree of internal segregation (20th generation of the simulation) as a function of the changes in the rate of residential mobility.

increase in the degree of segregation within the minority group; however, this effect is not directly proportional to the increase in the rate. This can be seen in a comparison of the results of the three simulations. Between the process with an $\mathrm{Mp}=0.3$ mobility rate and the process with an $\mathrm{Mp}=0.6$ per generation, no significant difference was found; in fact, sometimes the degree of segregation is greater in the case of the $\mathrm{Mp}=0.3$ mobility rate than in the case of the $\mathrm{Mp}=0.6$ mobility rate per generation.
The reason for this is the fact that increasing residential mobility has two opposite effects: a possibility of more effective utilization of time, but also an increase in the "packaging capacity" constraint (a constraint that derives from states in which large numbers of agents act at the same time and in the same space). The presence of this constraint has a deleterious effect on the level of coordination of actions and on the possibility of agents to direct their actions. Evidence of this trend comes from an examination of the effect of a change in the residential mobility rate in a wide range on the degree of residential segregation (Fig. 7(b)).

The analysis shows that the rate of minority population diffusion into areas inhabited by the majority population, and residential mobility within these areas, have a significant impact on the degree of segregation in the residential distribution. Together, they create the "environment" in which the residential segregation process occurs. In terms of space-time, the effects of demographic changes are realized in the form of a reduction or increase in the level of space and time resources. Since these resources are used in the choice of places of residence, the level of these resources must have an impact on the majority-minority residential distribution as well as on the distributions nested within the minority group's spatial pattern. However, it should be noted that demographic processes, such as those examined here, do not determine the degree of segregation and the constraints on the actions of agents. They serve only as conditions for action. They have the effect of intensifying or weakening the constraints responsible for the gap between the actions of agents and the aggregate distribution that the degree of residential segregation reflects here.

The gap between the behavior and intentions of agents and their results characterizes every complex system that emerges from a "self-organization" process (Allen et al., 1985; Portugali et al., 1994), and the residential segregation process examined here is no exception. The process occurs by means of a large number of spatio-temporal behaviors of discrete agents, in a manner that is not organized. 
This gap can be avoided only by preventing action from existing, i.e., only a state in which the system is organized in an "artificial" manner, would enable the creation of a residential distribution characterized by an ideal order, thereby preventing the impact of demographic processes which have the effect of intensifying or weakening constraints. However, the residential segregation process is not an organized one. It is one that becomes organized, which is the central feature of the "self-organization" process; a process without a planner or controller deciding where and when to "move" agents to create an integrated or segregated form. The process emerges from the agents, without their intent, as their is no collective goal. The process is composed of the actions of agents with their own goal. This explains why complete understanding of the self-organization process cannot be attained by simply analyzing the behavior of agents and the product of their behavior. In this type of process, it is necessary to investigate both the processes deriving from the context of the action, and the constraints deriving from processes that influence the space and time resources provided for the performance of the action, such as the demographic processes examined here.

\section{CONCLUSIONS}

Examination of spatial pattern diffusion and residential mobility in the context of residential segregation demonstrates the possibilities of using time-space concepts as analytical tools and a simulation model as a means for heuristic investigation. The translation of the phenomenon being examined by time-space concepts enables a common language for components and their relationship between them; in the present instance, between residential segregation, the minority group's spatial pattern and its form of diffusion, and demographic processes. Moreover, it also enables a common language between these components associated with the group level, and the actions of discrete individuals. The simulation model then enables use of this language i.e., investigation at the operative level. Since the agent-based simulation model constructed here allows the intentions and behavior of individuals to be attributed to space and time only (when to change place of residence and where to go), space and time are the only constraints to be taken into account. This enables "pure" investigation of the effects of demographic changes - since their full effect will be solely in terms of the enlargement or reduction of space and time resources. The combination of a conceptual framework and a simulation model enables abstraction at both the theoretical and operative levels, necessary for processing components that have an internal relationship; from the components in a minority group's form of diffusion - the geographic area size and rate of change in each geographic unit - to the relationship between the actions of individuals and the residential distribution as a whole.

The methodology presented here is not in principle limited to the study of demographic processes. It is adaptable for the examination of a wide range of urban processes, such as, for example, changes in the apartments inventory applicable to a minority group, changes in the built-up urban environment, etc. These urban processes have a direct bearing on demographic processes, and as such, as shown in the present study, an indirect bearing on residential segregation.

\section{References}

Allen, P., Sanglier, M., Engelen, G. and Boon, F., 1985, Towards a new synthesis in the modeling of evolving complex systems, Environment and Planning $B, \mathbf{1 2}, 65-84$.

Benenson, I. and Portugali, J., 1997, Agent-based simulations of a city dynamic in a Gis, Environment and Planning B, 24, 205-218.

Benno, W., 1993, Society, Action and Space, Routledge, London.

Boal, F.W., 1987, Segregation, In: M. Pasion (ed.), Social Geography: Progress and Prospect, Croom Helm, Beckenham.

Carlstein, C., 1982, Time Resources, Society and Ecology, Lund Studies in Geography.

Couclelis, H., 1985, Cellular worlds: A framework for modeling micro-macro dynamics, Environment and Planning A, 17, 585-596.

Couclelis, H., 1988, Of mice and men: what rodent population can teach us about complex spatial dynamics, Environment and Planning A, 20, 99-109. 
Demongeot, J., Goles, E. and Tchuente, M. (eds.), 1985, Dynamical Systems and Cellular Automata, Academic Press, London.

Dibble, C., 1996, Theory in a complex world: agent-based simulations of geographic systems. GeoComputation 96, 1st International Conference on GeoComputation, University of Leeds, Leeds.

Flowerdew, R., 1978, The role of time in residential choice models, In: Timing Space and Spacing Time, Vol. 2, Edward Arnold, London.

Giddens, A., 1984, The Constitution of Society: Outline of The Theory of Structuration, University of California Press, Berkeley.

Godfrey, B.J., 1988, Neighborhoods in Transition, University of California Publication in Geography, CA.

Hagerstrand, T., 1967, Innovation Diffusion as a Spatial Process, University of Chicago Press, Chicago.

Hagerstrand, T., 1970, What about people in regional science?, Papers and Proceedings of the Regional Science Association, Vol. 24, pp. 7-24.

Hendelman, D., 1977, The organization of ethnicity, Ethnic Groups, 1, 187-200.

Langton, G., 1986, Studying artificial life with cellular automata, Physica, 22D, 120-149.

Lierop, W.V., 1986, Spatial Interaction Modelling and Residential Choice Analysis, Gower, Vermont.

Manneville, P., Boccara, N., Vichniac, G.Y. and Bidaux, R. (eds.), 1989, Cellular Automata and Modeling of Complex Physical System, Springer-Verlag, Berlin.

Morrill, R.L., 1965, The Negro ghetto: Problems and alternatives, Geographical Review, 55, 339-369.

Morrill, R.L., 1970, The shape of diffusion in space and time, Economic Geography, 259-268.
Omer, I., 1996, Ethnic residential segregation as a structuration process. Unpublished Ph.D. Thesis, Tel-Aviv University, TelAviv.

Openshaw, S., 1995, Commentary: Human system modeling as a new grand challenge area in science, Environment and Planning A, 27, 159-164.

Phipps, M., 1989, Dynamical behavior of cellular automata under the constraint of neighborhood coherence, Geographical Analysis, 21(3), 197-215.

Portugali, J., Benenson, I. and Omer, I., 1994, Socio-spatial residental dynamics, stability and instability within a selforganizing city, Geographical Analysis, 26(4), 321-340.

Portugali, J., Benenson, I. and Omer, I., 1997, Spatial cognitive dissonance and sociospatial emergence in a self-organizing city, Environment and Planning B, 24, 263-285.

Sayer, A., 1984, Method and Social Science, Hutchinson, London.

Tobler, W.R., 1979, Cellular geography, In: S. Gale and G. Olsson (eds.), Philosophy in Geography, Reidel, Dordrecht.

Weidlich, W., 1987, Synergetics and social science, In: R. Graham and A. Wunderlin (eds.), Lasers and Synergetics, Springer-Verlag.

White, R., Engelen, G. and Uljee, I., 1997, The use of constrained cellular automata for high-resolution modeling of urban land-use patterns, Environment and Planning B, 24, $323-343$.

Woods, R.I., 1980, Spatiotemporal models of ethnic segragation and their implications for housing policy, Environment and Planning A, 13, 1415-1433.

Wu, F., 1996, A linguistic cellular automata simulation approach for sustainable land development in a fast growing region, Comput., Environ. Urban Systems, 20, 367-378. 


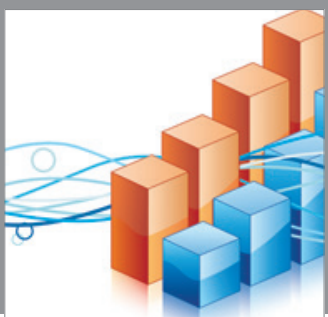

Advances in

Operations Research

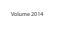

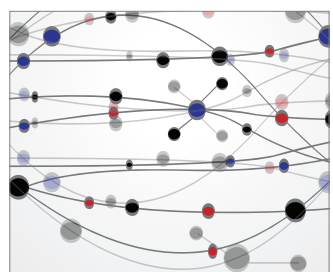

\section{The Scientific} World Journal
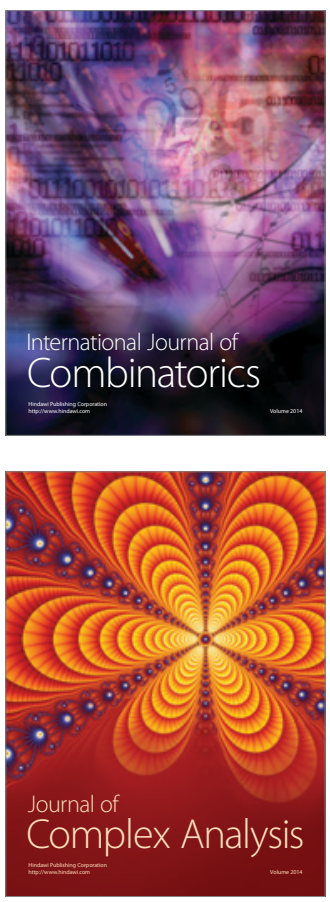

International Journal of

Mathematics and

Mathematical

Sciences
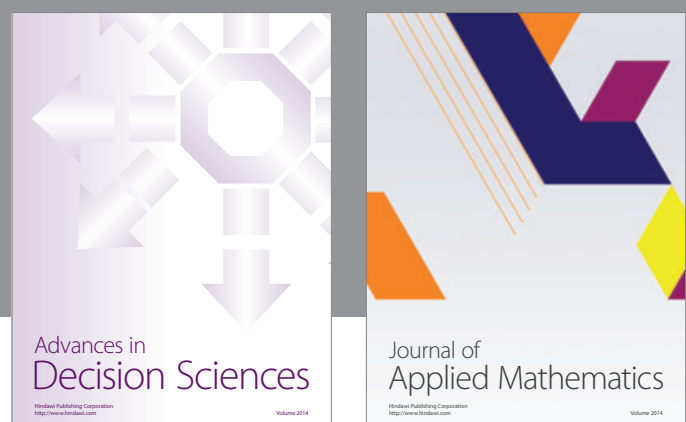

Journal of

Applied Mathematics
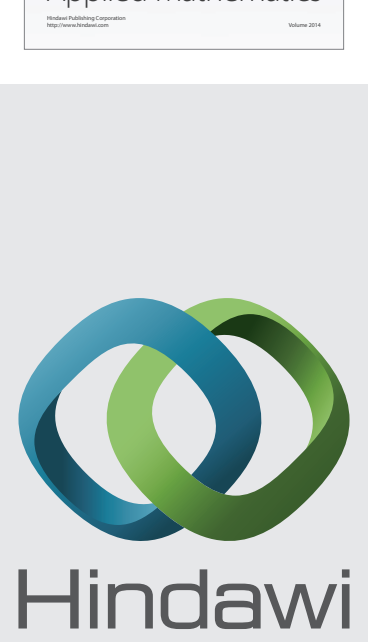

Submit your manuscripts at http://www.hindawi.com
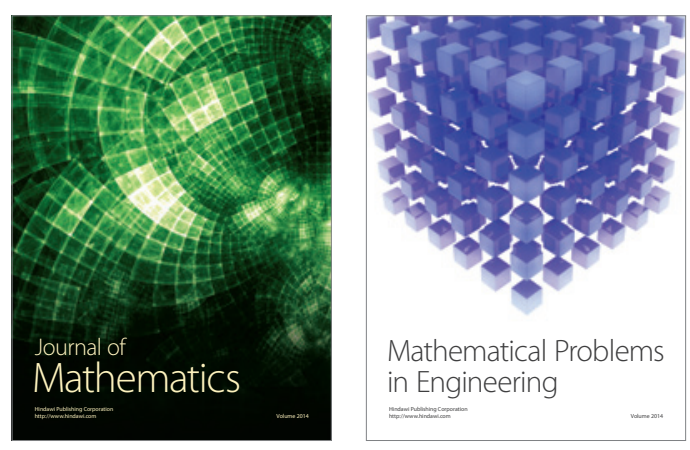

Mathematical Problems in Engineering
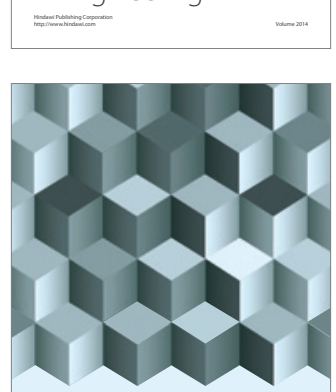

Journal of

Function Spaces
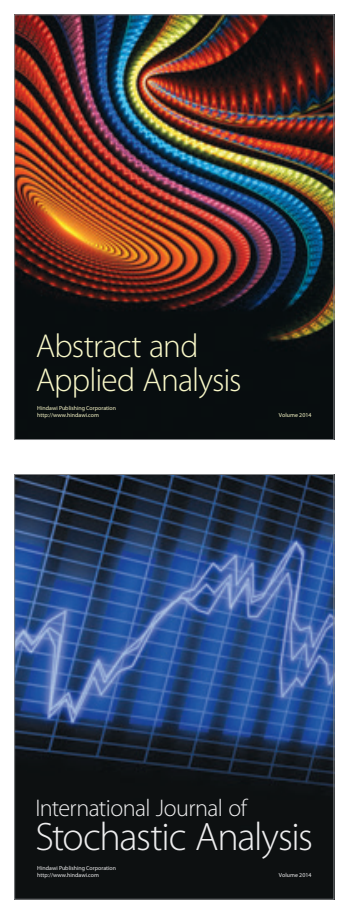

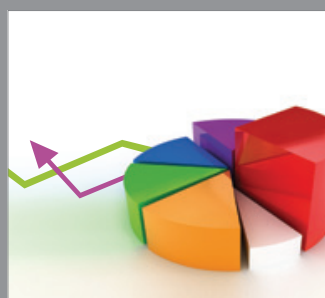

ournal of

Probability and Statistics

Promensencen
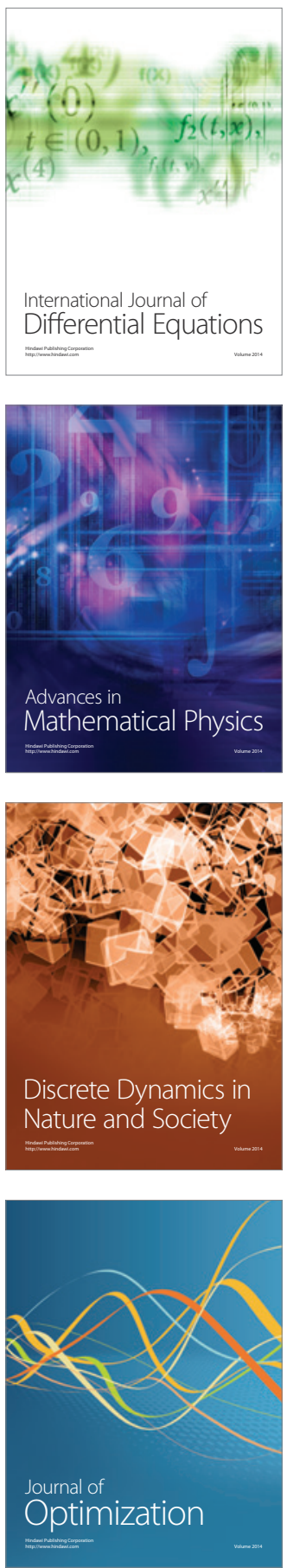